\title{
Effect of onion flavonoids on colorectal cancer with hyperlipidemia: an in vivo study
}

This article was published in the following Dove Press journal:

OncoTargets and Therapy

8 January 2014

Number of times this article has been viewed

\section{Yongshan $\mathrm{He}^{1, *}$ \\ Heiying Jin ${ }^{1, *}$ \\ Wei Gong ${ }^{2, *}$ \\ Chunxia Zhang' \\ Acheng Zhou'}

'National Center of Colorectal Surgery, Third Affiliated Hospital of Nanjing University of Traditional Chinese Medicine, Nanjing, People's Republic of China; ${ }^{2}$ Department of Surgery, Jiangyin Hospital of Traditional Chinese Medicine, Jiangyin, People's Republic of China

*These authors contributed equally to this work
Correspondence: Heiying Jin National Center of Colorectal Surgery, Third Affiliated Hospital of Nanjing University of Traditional Chinese Medicine, I Jinling Road, Nanjing, Jiangsu 210001 , People's Republic of China

Tel +86 I33 82793886

Email jinheiying@hotmail.com
Objectives: This study aims to find the effect of onion's extraction on the colorectal cancer with hyperlipidemia.

Method: We established a hyperlipidemia-subcutaneously heterotopic colorectal cancer orthotopic transplant model and fed mice a high fat diet and performing transplantation. Animal models were treated with capecitabine and/or simvastatin and low-, middle-, high- dose of onion's extraction and both tumor growth rate and blood lipid levels were monitored.

Results: We found that colorectal cancer in onion's extraction groups was significantly inhibited, and the effect of high dose of onion's extraction was equivalent to capecitabine. Onion's extraction effectively decreased levels of apoB and TC.

Conclusion: Our study established a hyperlipidemia colon tumor model involving subcutaneous colon translocation and orthotopic transplantation, this model was an ideal research model for mutual influence of hyperlipidemia and colorectal cancer. Onion's extraction could inhibit the proliferation of colorectal cancer; the function of the high-dose of onion's extraction was fairly to capecitabine, which provided a new direction in protecting and treating colorectal cancer.

Keywords: colorectal cancer, hyperlipidemia, onion flavonoids, capecitabine, simvastatin

\section{Introduction}

Onion (Allium cepa L.) has been used for centuries for its pungency, flavoring value, and medicinal properties. The bulb of onion is used medicinally, and has been consumed as seasoning food for many centuries. Phytochemical research has proved that onion is rich in flavonols and organosulfur compounds, which have exhibited tumor-inhibitory properties in laboratory studies. ${ }^{1}$ Galeone et $\mathrm{al}^{2}$ used data from an integrated network of Italian and Swiss case-control studies to analyze the relation between frequency of onion and garlic use and cancer at several sites. They found that consumption of onions varied between 0-14 and 0-22 portions/ week among cases and controls, respectively. The multivariate odds ratios for the highest category of onion and garlic intake were, respectively, 0.16 and 0.61 for cancer of the oral cavity and pharynx, 0.12 and 0.43 for esophageal cancer, 0.44 and 0.74 for colorectal cancer, 0.17 and 0.56 for laryngeal cancer, 0.75 and 0.90 for breast cancer, 0.27 and 0.78 for ovarian cancer, 0.29 and 0.81 for prostate cancer, and 0.62 and 0.69 for renal cell cancer. They concluded that there is an inverse association between the frequency of use of allium vegetables and the risk of several common cancers. Other epidemiological investigation indicates that 
onion plays a role in preventing gastric, colorectal, and prostatic cancers. ${ }^{3}$ Though onion flavonoids may play a role in preventing colorectal neoplasm, there is not enough evidence to support this conclusion. ${ }^{4}$ Our previous study also demonstrated that flavonoids inhibit the proliferation of LoVo cells, SW480 cells, HT-29 cells, and HCT-8 cells in a time- and concentration-dependent manner. ${ }^{5}$

Studies have showed that hyperlipidemia is associated with colorectal adenomas. ${ }^{6,7}$ Can onion prevent colorectal cancer as well as alleviate hyperlipidemia? Lee et $\mathrm{al}^{8}$ reported that food containing flavonoids can decrease the incidence of cardiovascular disease and metabolic disease in male smokers, and Movahedian et $\mathrm{al}^{9}$ suggested that onion alleviates hypercholesterolemia in rabbits. However, little research has been done on the role of onion in colorectal cancer, especially under conditions of abnormal lipid metabolism.

In this study, hyperlipidemic subcutaneous heterotopic colorectal cancer fluorescent orthotopic transplant mice were treated with aqueous-extracted onion flavonoids. Tumor growth and the effect of flavonoids on hyperlipidemia were observed. The role of onion extract in hyperlipidemic colorectal cancer was confirmed, and results of this research provide a foundation for clinical research.

\section{Materials and methods}

\section{Animals}

Specific pathogen-free BALB/C nu/nu mice ranging in mass from 20 to $22 \mathrm{~g}$ and aged from 4 to 6 weeks were provided by The Experimental Animal Center, Yangzhou University. All animal experiments were approved by the Ethics Committee of Experimental Animals.

\section{Preparation of fat emulsion}

The fat emulsion was prepared as follows: $20 \mathrm{~g}$ lard, $5 \mathrm{~g}$ cholesterol, and $10 \mathrm{~g}$ Tween 80 was dissolved in $50 \mathrm{~mL}$ water. Mice were intragastrically administered $1 \mathrm{~mL} / 10 \mathrm{~g}$ of the emulsion each day.

\section{Detection of blood lipid levels}

All detection reagents for total cholesterol (TC), triglyceride (TG), high-density lipoprotein (HDL), and low-density lipoprotein (LDL) were obtained from Biosino Bio-Technology and Scientific (Beijing, People's Republic of China). The reagent to detect lipoprotein(a) (Lpa) was purchased from Ausbio (Yantai, People's Republic of China). Reagents for apolipoprotein A (apoA) and apoB were obtained from
Shanghai Source Leaf Biological Technology (Shanghai, People's Republic of China).

\section{Extraction and detection of onion flavonoids}

As described previously, ${ }^{5}$ onion flavonoids were extracted using an aqueous extraction method and further concentrated.

\section{Establishment of the hyperlipidemia model}

A total of 100 nude mice were randomly divided into ten groups ( $\mathrm{n}=10$ in each group), and body weight was comparable in all groups $(P>0.05)$. Eight groups were randomly selected to receive a high-fat diet, and the other two groups received normal diets (control groups). The high-fat diet groups were intragastrically administered fat emulsions based on their weights. Four weeks later, one of the high-fat diet groups and one of the normal diet groups were randomly selected as an experimental group, and the other control groups were used to establish the model. Blood was collected from the eyes after fasting for 12 hours. The blood was centrifuged at 3,500-4,000 rpm for 10 minutes. TC and TG levels were detected using enzymatic methods, and HDL, LDL, and Lpa were directly measured. ApoA and apoB were detected using an enzymelinked immunosorbent assay. The normal value of lipid was defined as the $95 \%$ confidence interval of the lipid value in the normal diet group. When the TC, TG, or LDL was higher than the normal value, the mice were diagnosed as hyperlipidemic. ${ }^{10}$

\section{Establishment of the hyperlipidemia subcutaneous heterotopic colorectal cancer fluorescent orthotopic transplant model}

As previously described, ${ }^{11}$ the mice in the remaining two groups were used to establish the subcutaneous heterotopic colorectal cancer fluorescent orthotopic transplant model. Fluorescent tumors could be seen by the tenth day after transplant under a fluorescent light. Tumor volumes were calculated, and the mice in the high-fat diet groups continued to receive the intragastric fat emulsion. Tumor volumes and growth rates were obtained as follows: volume $\left(\mathrm{mm}^{3}\right)=1 / 2 \mathrm{ab}^{2}$ ( $a$, major axis; $b$, minor axis), and growth rate $=$ (measured volume at each time point - beginning volume)/beginning volume. 


\section{Experimental groups}

Tumors in all groups were detectable within 2 weeks after tumor-tissue transplant, when the tumor size was initially measured. The mice in the standard diet with capecitabine (SDT-CAP) group were intragastrically administered capecitabine (Xeloda; Roche, Basel, Switzerland) at a dose of $359 \mathrm{mg} / \mathrm{kg} / \mathrm{day}$ for 5 consecutive days. ${ }^{12}$ The mice in the high-fat diet group were randomly divided into seven groups: fat food with saline (FAT-SAL), fat food with capecitabine (FAT-CAP), fat food with simvastatin (FAT-SIM), FAT-SIM and capecitabine (FAT-SIM+CAP), fat diet and low-dose onion flavonoids (FAT-ONION-L), fat diet and middle-dose onion flavonoids (FAT-ONION-M), and fat diet and high-dose onion flavonoids (FAT-ONION-H). Mice in FAT-SAL were intragastrically administered saline at a dose of $359 \mathrm{mg} / \mathrm{kg} /$ day. The mice in the FAT-CAP group intragastrically received capecitabine $(359 \mathrm{mg} / \mathrm{kg} /$ day $)$. Mice in the FAT-SIM group were treated with simvastatin at a dose of $6.6 \mathrm{mg} / \mathrm{kg} /$ day $^{13}$ intragastrically. Mice in FAT-SIM+CAP received intragastric capecitabine and simvastatin at doses of $359 \mathrm{mg} / \mathrm{kg} /$ day and $6.6 \mathrm{mg} / \mathrm{kg} /$ day, respectively. Mice in the FAT-ONION-L, FATONION-M, and FAT-ONION-H groups were treated with low, middle, and high doses of onion flavonoids, respectively. The mice were treated with drugs for 3 weeks. Two weeks later, the mice were fasted for 12 hours before collecting blood from their eyes. Serum was obtained using centrifugation. The mice were killed, and their tumors were harvested. The experimental groups are shown in Table 1.

\section{Statistical analysis}

Data were processed with SPSS 17.0 (IBM, Armonk, NY, USA), and quantitative data were expressed as means \pm standard deviation; $t$-tests were also applied when appropriate. Repeatedmeasure data such as tumor volume and tumor-increase rate were evaluated with a general linear model.
Table 2 Blood lipid level in normal diet and high-fat diet groups

\begin{tabular}{lccccc}
\hline Lipid & $\begin{array}{l}\text { Normal diet } \\
\text { group (normal } \\
\text { value } \mathbf{n = 1 0 )}\end{array}$ & \multicolumn{4}{l}{ High-fat diet group $(\mathbf{n}=\mathbf{1 0})$} \\
\cline { 2 - 6 } & Mean \pm SD & High & Normal & Low \\
\hline TC & $2.75 \pm 0.23$ & $3.21 \pm 0.51$ & 9 & I & 0 \\
TG & $1.50 \pm 0.47$ & $1.52 \pm 0.35$ & 0 & 9 & I \\
HDL & $1.73 \pm 0.11$ & $2.19 \pm 0.32$ & 7 & 3 & 0 \\
LDL & $0.19 \pm 0.02$ & $0.21 \pm 0.07$ & 6 & 2 & 2 \\
Lpa & $165.30 \pm 27.20$ & $170.50 \pm 24.70$ & 2 & 8 & 0 \\
apoA & $729.17 \pm 143.17$ & $1086.14 \pm 144.68$ & 8 & 2 & 0 \\
apoB & $280.98 \pm 58.95$ & $357.70 \pm 95.83$ & 4 & 6 & 0 \\
\hline
\end{tabular}

Abbreviations: TC, total cholesterol; TG, triglyceride; $\mathrm{HDL}$, high-density lipoprotein; LDL, low-density lipoprotein; Lpa, lipoprotein(a); apoA, apolipoprotein A; SD, standard deviation.

\section{Results}

\section{Detection of blood lipids in normal diet and high-fat diet groups}

In Table 2, the normal value of blood lipid is summarized in the left column. Compared with the normal value, in the high-fat diet group, there were five of ten animals with high TC and LDL, four with high TC, and one with high LDL. There was no animal with high TG. According to the hyperlipidemia diagnostic criteria, all animals were diagnosed as hyperlipidemic. The values of HDL, Lpa, apoA, and apoB are summarized in Table 2.

\section{Comparison of tumor volume and growth rate between normal diet and high-fat diet groups}

Mice in the FAT-SAL group had the fastest tumor-growth rate, and this rate was significantly faster than the tumor-growth rate in other groups, except the FAT-SIM group, which meant the negative control with the highest tumor-growth rate and simvastatin did not influence the growth of tumors.

Tumor volume in the FAT-ONION groups was lower than that in the FAT-SAL and FAT-SIM, groups and the difference

Table I Experimental groups $(n=80)$

\begin{tabular}{|c|c|c|c|c|c|}
\hline Group & $\mathbf{n}$ & Experimental compounds & $\begin{array}{l}\text { Dosage per } \\
\text { day (mg/kg) }\end{array}$ & $\begin{array}{l}\text { Administration } \\
\text { time (weeks) }\end{array}$ & $\begin{array}{l}\text { Method of } \\
\text { administration }\end{array}$ \\
\hline SDT-CAP & 10 & Capecitabine & 359 & 3 & IG \\
\hline FAT-SAL & 10 & Saline & 359 & 3 & IG \\
\hline FAT-CAP & 10 & Capecitabine & 359 & 3 & IG \\
\hline FAT-SIM & 10 & Simvastatin & 6.6 & 3 & IG \\
\hline FAT-SIM+CAP & 10 & Capecitabine + simvastatin & $359+6.6$ & 3 & IG \\
\hline FAT-ONION-L & 10 & Onion flavonoids (low dose) & 100 & 3 & IG \\
\hline FAT-ONION-M & 10 & Onion flavonoids (middle dose) & 200 & 3 & IG \\
\hline FAT-ONION-H & 10 & Onion flavonoids (high dose) & 300 & 3 & IG \\
\hline
\end{tabular}

Abbreviation: IG, intragastric; SDT-CAP, standard diet with capecitabine; FAT-SAL, fat food with saline; FAT-CAP, fat food with capecitabine; FAT-SIM, fat food with simvastatin; FAT-SIM+CAP, fat food with simvastatin and capecitabine; FAT-ONION-L, fat diet and low-dose onion flavonoids; FAT-ONION-M, fat diet and middle-dose onion flavonoids; FAT-ONION-H, fat diet and high-dose onion flavonoids. 
Table 3 Tumor volume in each group $\left(\mathrm{mm}^{3}\right)$

\begin{tabular}{|c|c|c|c|c|c|c|}
\hline Group & 0 & Week I & Week 2 & Week 3 & Week 4 & Week 5 \\
\hline SDT-CAP & $85.46 \pm 18.92$ & $137.60 \pm 82.57$ & $257.30 \pm 186.54$ & $478.5 \pm 304.56$ & $693.79 \pm 443.21$ & $823.30 \pm 370.68$ \\
\hline FAT-SAL & $85.29 \pm 7.11$ & $400.89 \pm 132.95$ & $703.95 \pm 343.40$ & $\mathrm{I}, 057.25 \pm 5 \mathrm{I} 2.40$ & I,39|.79 $\pm 8 \mid 8.97$ & $\mathrm{I}, 889.07 \pm 830.96$ \\
\hline FAT-CAP & $84.78 \pm 15.30$ & $|21.75 \pm 7| .93$ & $208.49 \pm 187.02$ & $253.12 \pm 252.49$ & $307.53 \pm 337.59$ & $379.40 \pm 360.07$ \\
\hline FAT-SIM & $86.52 \pm 26.59$ & $355.22 \pm 198.16$ & $549.85 \pm 330.90$ & $758.7 I \pm 442.13$ & $\mathrm{I}, 096.64 \pm 448.92$ & $\mathrm{I}, 649.23 \pm 597.89$ \\
\hline FAT-SIM+CAP & $83.81 \pm 19.89$ & $82.28 \pm 77.08$ & $|4| .04 \pm \mid 55.33$ & $238.48 \pm 216.99$ & $321.96 \pm 237.67$ & $449.24 \pm 301.65$ \\
\hline FAT-ONION-L & $87.88 \pm 29.71$ & $246.86 \pm 164.79$ & $46 \mid .39 \pm 25$ I.24 & $647.7 I \pm 260.31$ & $835.57 \pm 249.19$ & $1,051.25 \pm 204.67$ \\
\hline FAT-ONION-M & $86.47 \pm 29.41$ & $167.75 \pm 94.80$ & $387.38 \pm 205.42$ & $573.37 \pm 326.29$ & $831.65 \pm 425.64$ & $961.19 \pm 555.97$ \\
\hline FAT-ONION-H & $84.02 \pm 21.14$ & | $33.67 \pm 48.46$ & $234.22 \pm 106.98$ & $373.14 \pm 161.78$ & $554 .|8 \pm 25| .36$ & $622.95 \pm 353.23$ \\
\hline
\end{tabular}

Abbreviations: SDT-CAP, standard diet with capecitabine; FAT-SAL, fat food with saline; FAT-CAP, fat food with capecitabine; FAT-SIM, fat food with simvastatin; FATSIM+CAP, fat food with simvastatin and capecitabine; FAT-ONION-L, fat diet and low-dose onion flavonoids; FAT-ONION-M, fat diet and middle-dose onion flavonoids; FAT-ONION-H, fat diet and high-dose onion flavonoids.

was significant $(P<0.05)$, which implied that onion flavonoids can inhibit tumor growth. Tumor-growth rate in the FATONION-L and FAT-ONION-M groups was higher than that in other groups and FAT-ONION-H was lower than in other groups, but the difference was not statistically significant $(P>0.05)$. The tumor growth rates in FAT-ONION-H group were as low as those in FAT-CAP, FAT-SIM+CAP and SDTCAP groups, which meant that in this study, the FAT-ONION-H has a similar antitumor effect compared to capecitabine (Tables 3, 4 and Figure 1). All growth rates were in accordance with tumor volumes (Tables 5, 6 and Figure 2).

\section{Comparison of tumor weights between normal diet and high-fat diet groups}

After 5 weeks, the mice were killed, and tumors were harvested and weighed. Tumor weight showed the same tendency as tumor volume and tumor-growth rate. The FAT-SAL and FAT-SIM groups had the highest tumor weight of all the groups. Among other groups, except for the FAT-SAL and FAT-SIM groups, the tumor weight was not statistically significant $(P>0.05)$. All data are shown in Table 7 and Figure 3.

\section{Detection of blood lipids}

Three weeks after treatment, TC increased in the FATSAL group, while TC was decreased in the other seven groups. This decrease was most pronounced for mice in the FAT-SIM+CAP group. The TC level in all these groups decreased by week 5, except in the FAT-ONION-M and FAT-ONION-H groups. Moreover, LDL significantly increased in SDT-CAP, FAT-SAL, and FAT-CAP, and decreased in FAT-SIM, FAT-SIM+CAP, FAT-ONION-L, FAT-ONION-M, and FAT-ONION-H. However, blood lipid levels in the FAT-SIM, FAT-ONION-L, FAT-ONION-M, and FAT-ONION-H groups at week 5 were higher than those in week 3. TG, Lpa and apoA levels in these groups decreased by weeks 3 and 5. The HDL and apoB levels in the FAT-ONION-L, FAT-ONION-M, and FAT-ONION-H groups decreased at week 3 and were increased at week 5. TC, HDL, and apoB levels in FAT-ONION-L, FAT-ONION-M, and FAT-ONION-H were remarkably lower than in FAT-SAL at the beginning and week 3. Furthermore, TG levels in FAT-SAL, FAT-CAP, and FATSIM markedly increased at week 5 . All data are shown in Table 8 .

Table 4 Comparison of tumor volume among these groups with general linear model ( $P$-value)

\begin{tabular}{lllllll}
\hline Group & SDT-CAP & FAT-SAL & FAT-CAP & FAT-SIM & $\begin{array}{l}\text { FAT- } \\
\text { SIM+CAP }\end{array}$ & $\begin{array}{l}\text { FAT- } \\
\text { ONION-L }\end{array}$ \\
\hline FAT-SAL & 0.001 & & & & \\
FAT-CAP & 0.626 & 0.000 & & & & \\
ONION-M
\end{tabular}

Abbreviations: SDT-CAP, standard diet with capecitabine; FAT-SAL, fat food with saline; FAT-CAP, fat food with capecitabine; FAT-SIM, fat food with simvastatin; FATSIM+CAP, fat food with simvastatin and capecitabine; FAT-ONION-L, fat diet and low-dose onion flavonoids; FAT-ONION-M, fat diet and middle-dose onion flavonoids; FAT-ONION-H, fat diet and high-dose onion flavonoids. 
Table 5 Tumor growth rate in each group

\begin{tabular}{lcccrc}
\hline Group & Week I & Week 2 & Week 3 & Week 4 & Week 5 \\
\hline SDT-CAP & $0.58 \pm 0.76$ & $1.95 \pm 1.83$ & $4.37 \pm 2.71$ & $6.45 \pm 3.50$ & $7.74 \pm 2.74$ \\
FAT-SAL & $3.76 \pm 1.29$ & $7.08 \pm 3.04$ & $11.21 \pm 4.80$ & $14.53 \pm 8.73$ & $19.65 \pm 8.79$ \\
FAT-CAP & $0.43 \pm 0.78$ & $1.41 \pm 2.08$ & $1.96 \pm 3.01$ & $2.19 \pm 3.03$ & $3.05 \pm 3.26$ \\
FAT-SIM & $3.78 \pm 3.92$ & $5.97 \pm 5.56$ & $8.51 \pm 6.82$ & $12.91 \pm 7.95$ & $17.90 \pm 7.49$ \\
FAT-SIM+CAP & $-0.07 \pm 0.80$ & $0.53 \pm 1.30$ & $1.66 \pm 1.84$ & $2.89 \pm 2.59$ & $1.41 \pm 3.11$ \\
FAT-ONION-L & $1.58 \pm 1.06$ & $4.04 \pm 1.78$ & $6.45 \pm 1.76$ & $9.84 \pm 4.33$ & $12.83 \pm 4.38$ \\
FAT-ONION-M & $0.98 \pm 0.94$ & $3.67 \pm 2.42$ & $5.71 \pm 4.30$ & $3.42 \pm 7.07$ & $14.35 \pm 12.24$ \\
FAT-ONION-H & $0.66 \pm 0.68$ & $1.93 \pm 1.45$ & $3.69 \pm 2.17$ & $6.58 \pm 3.79$ & $7.45 \pm 4.78$ \\
\hline
\end{tabular}

Abbreviations: SDT-CAP, standard diet with capecitabine; FAT-SAL, fat food with saline; FAT-CAP, fat food with capecitabine; FAT-SIM, fat food with simvastatin; FATSIM+CAP, fat food with simvastatin and capecitabine; FAT-ONION-L, fat diet and low-dose onion flavonoids; FAT-ONION-M, fat diet and middle-dose onion flavonoids; FAT-ONION-H, fat diet and high-dose onion flavonoids.

\section{Tumor volume after treatment}

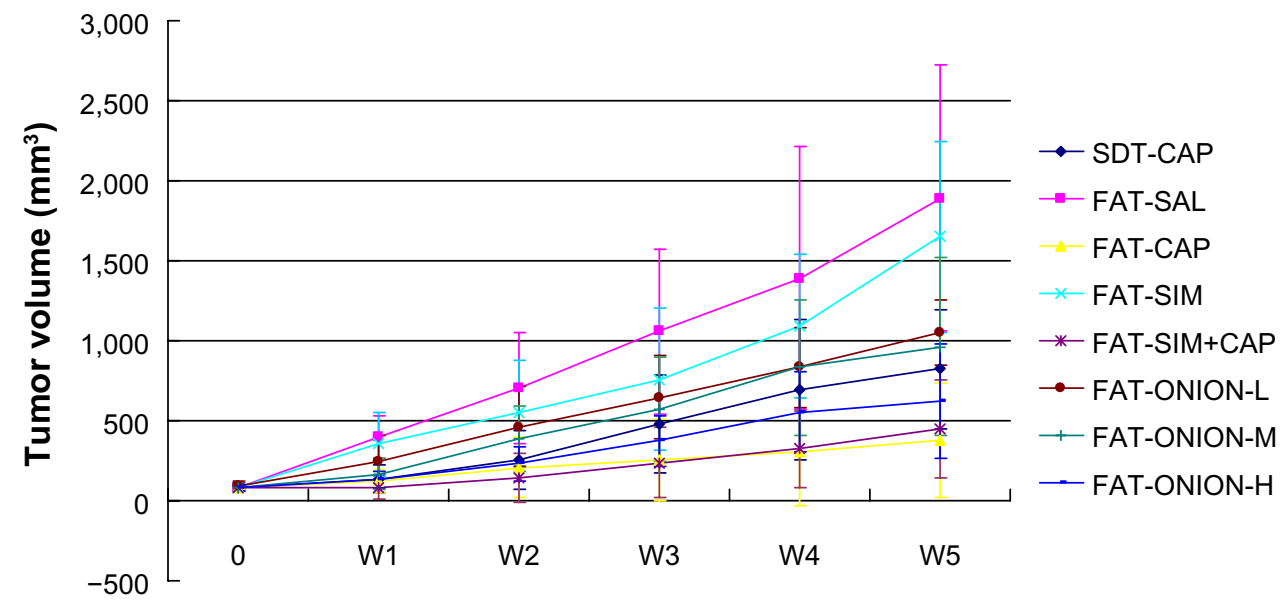

Time after treatment

Figure I Tumor volume after treatment. Mice in the FAT-SAL group had the fastest tumor-growth rate, and this rate was significantly faster than the tumor-growth rate in other groups except the FAT-SIM group. Additional analysis showed that the tumor-growth rates in mice in the FAT-SIM group, while markedly lower than those in the FAT-SAL group, was higher than those in the SDT-CAP, FAT-CAP, FAT-SIM, FAT-SIM+CAP, FAT-ONION-L, FAT-ONION-M, and FAT-ONION-H groups. The tumorgrowth rates in the SDT-CAP group were lower than those in the FAT-ONION-L and FAT-ONION-M groups and higher than those in the FAT-CAP, FAT-SIM+CAP, and FAT-ONION-H groups $(P>0.05)$. Tumors in mice in the FAT-SIM+CAP group displayed the slowest growth rate; this growth rate was slightly lower than the growth rate in the FAT-ONION-H and FAT-CAP groups $(P>0.05)$, and remarkably lower than that in the FAT-ONION-L and FAT-ONION-M groups $(P<0.05)$. No significant difference was observed among the FAT-ONION-L, FAT-ONION-M, and FAT-ONION-H groups. However, the fastest and slowest growth rates were present in the FAT-ONION-L group and FAT-ONION-H group, respectively.

Abbreviations: SDT-CAP, standard diet with capecitabine; FAT-SAL, fat food with saline; FAT-CAP, fat food with capecitabine; FAT-SIM, fat food with simvastatin; FATSIM+CAP, fat food with simvastatin and capecitabine; FAT-ONION-L, fat diet and low-dose onion flavonoids; FAT-ONION-M, fat diet and middle-dose onion flavonoids; FAT-ONION-H, fat diet and high-dose onion flavonoids; W, week.

Table 6 Comparison of growth rate (P-value)

\begin{tabular}{lllllll}
\hline Group & SDT-CAP & FAT-SAL & FAT-CAP & FAT-SIM & $\begin{array}{l}\text { FAT- } \\
\text { SIM+CAP }\end{array}$ & $\begin{array}{l}\text { FAT- } \\
\text { ONION-L }\end{array}$ \\
\hline FAT-SAL & 0.002 & & & & \\
FAT-CAP & 0.734 & 0.000 & & & & \\
ONION-M
\end{tabular}

Abbreviations: SDT-CAP, standard diet with capecitabine; FAT-SAL, fat food with saline; FAT-CAP, fat food with capecitabine; FAT-SIM, fat food with simvastatin; FATSIM+CAP, fat food with simvastatin and capecitabine; FAT-ONION-L, fat diet and low-dose onion flavonoids; FAT-ONION-M, fat diet and middle-dose onion flavonoids; FAT-ONION-H, fat diet and high-dose onion flavonoids. 
Table 7 Comparison of tumor weight

\begin{tabular}{lllllllll}
\hline Group & Weight $(\mathrm{g})$ & FAT-SAL & FAT-CAP & FAT-SIM & $\begin{array}{l}\text { FAT- } \\
\text { SIM+CAP }\end{array}$ & $\begin{array}{l}\text { FAT- } \\
\text { ONION-L }\end{array}$ & $\begin{array}{l}\text { FAT- } \\
\text { ONION-M }\end{array}$ & $\begin{array}{l}\text { FAT- } \\
\text { ONION-H }\end{array}$ \\
\hline SDT-CAP & $1.03 \pm 0.40$ & 0.024 & 0.011 & 0.395 & 0.047 & 0.686 & 0.224 & 0.123 \\
FAT-SAL & $1.59 \pm 0.74$ & - & 0.000 & 0.154 & 0.000 & 0.008 & 0.000 & 0.000 \\
FAT-CAP & $0.39 \pm 0.27$ & - & - & 0.001 & 0.528 & 0.031 & 0.124 & 0.375 \\
FAT-SIM & $1.24 \pm 0.53$ & - & - & - & 0.005 & 0.212 & 0.037 & 0.021 \\
FAT-SIM+CAP & $0.54 \pm 0.30$ & - & - & - & - & 0.112 & 0.367 & 0.753 \\
FAT-ONION-L & $0.93 \pm 0.15$ & - & - & - & - & - & 0.429 & 0.243 \\
FAT-ONION-M & $0.74 \pm 0.47$ & - & - & - & - & - & - & 0.618 \\
FAT-ONION-H & $0.62 \pm 0.14$ & - & - & - & - & - & - & - \\
\hline
\end{tabular}

Abbreviations: SDT-CAP, standard diet with capecitabine; FAT-SAL, fat food with saline; FAT-CAP, fat food with capecitabine; FAT-SIM, fat food with simvastatin; FATSIM+CAP, fat food with simvastatin and capecitabine; FAT-ONION-L, fat diet and low-dose onion flavonoids; FAT-ONION-M, fat diet and middle-dose onion flavonoids; FAT-ONION-H, fat diet and high-dose onion flavonoids.

\section{Discussion}

Hyperlipidemia is a common accompanying condition in patients with colorectal cancer, and it is a risk factor that may be associated with death in this population of cancer patients. ${ }^{14,15}$ The effect of hyperlipidemia on colorectal cancer has mainly been based on clinical observations. Hata et al established a metabolically aberrant transgenic mouse C57BL/KsJ-db/db-Apc (Min/+) (db/db-Min/+). ${ }^{16}$ They found that the occurrence of gastrointestinal tract adenomas in these transgenic mice was markedly higher than in adenomatous polyposis coli-deficient mice 15 weeks later. These findings indicate that hyperlipidemia can promote the occurrence of colorectal cancer. However, transgenic mice are difficult to obtain, and mouse polyp biology and reactivity differs from that of human polyps. Previous studies have investigated drugs used to treat abnormal lipid metabolism in hyperlipidemia in rats and mice. ${ }^{17}$ Based on these studies, nude mice were fed a high-fat diet. Four weeks later, all ten mice were diagnosed as hyperlipidemic according to the hyperlipidemia criteria. These findings suggest that the hyperlipidemia model can be established by feeding nude mice a high-fat diet. As previously described, ${ }^{11}$ the subcutaneous heterotopic colorectal cancer fluorescent orthotopic transplant model was established on the basis of hyperlipidemia in nude mice. The hyperlipidemia subcutaneous heterotopic colorectal cancer orthotopic transplant model was used in nude mice, and these mice presented with tumors; these results are in agreement with the previous study. ${ }^{11}$

\section{Tumor growth rate curves}

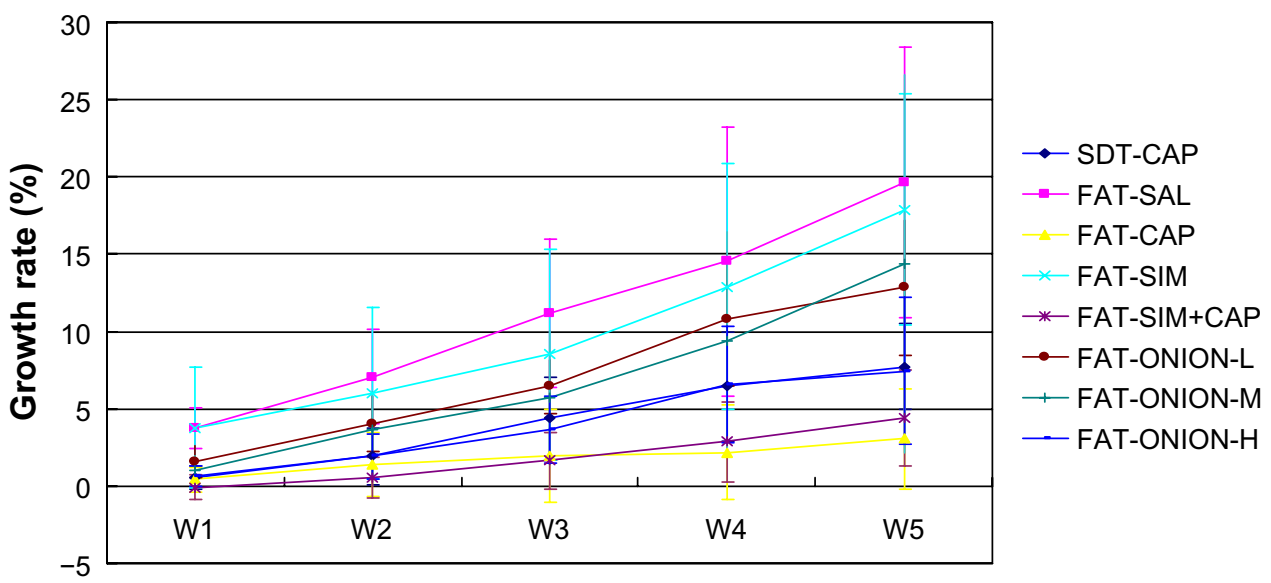

Time after treatment

Figure 2 Tumor growth-rate curves. Tumor growth-rate curves are similar to tumor-proliferation curves.

Abbreviations: SDT-CAP, standard diet with capecitabine; FAT-SAL, fat food with saline; FAT-CAP, fat food with capecitabine; FAT-SIM, fat food with simvastatin; FATSIM+CAP, fat food with simvastatin and capecitabine; FAT-ONION-L, fat diet and low-dose onion flavonoids; FAT-ONION-M, fat diet and middle-dose onion flavonoids; FAT-ONION-H, fat diet and high-dose onion flavonoids; W, week. 
Statins have been used to treat hyperlipidemia. Previous studies have also shown that statins play a role in preventing colorectal cancer. ${ }^{18-20}$ Recently, the underlying mechanisms by which statins inhibit colorectal cancer have been further investigated. ${ }^{13,21}$ Capecitabine was found to cause hyperlipidemia during chemotherapy in colorectal cancer, and thus application of antilipemic agents may elevate the effect of chemotherapy on colorectal cancer. ${ }^{22-24}$ Shimoyama deemed that statins may play a role in enhancing targeted drug effects during the treatment of colorectal cancer through decreasing the lipid level..$^{25}$

In our study, the FAT-SIM group showed a similar growth rate to FAT-SAL, which meant that the simvastatin did not show any anticancer effect on colorectal cancer. The FATSIM+CAP group showed a similar growth rate to the FATCAP group, which meant that the simvastatin did not have an enhancement effect on capecitabine. The reason may be that the duration of simvastatin use was too short to show a preventive effect on colorectal cancer.

In this study, the tumor-growth rates in FAT-ONION-L, FAT-ONION-M, and FAT-ONION-H were significantly lower than in FAT-SAL and FAT-SIM and higher than in SDT-CAP. These findings indicate that the role of onion flavonoids in hyperlipidemic colorectal cancer is similar to the role of capecitabine in normal colorectal cancer. The tumor-growth rates in FAT-CAP and FAT-SIM+CAP were markedly lower than in FAT-ONION-L and FAT-ONION-M. However, no significant difference was observed between FAT-ONION-H and FAT-CAP. The data suggested that the effect of high-dose onion flavonoids on inhibiting the tumor-growth rate was similar to that of capecitabine, and that they both play a role in the treatment of hyperlipidemic colorectal cancer.

Onion flavonoids dose-dependently inhibit tumor growth. The growth rate in FAT-ONION-L was remarkably lower than that in the FAT-ONION-H group. No significant difference was observed between FAT-ONION-M and FAT-ONION-H. It has been reported that onion flavonoids inhibit colorectal cancer cells in vitro. ${ }^{5,26,27}$ Our study demonstrated that onion flavonoids also markedly inhibit colorectal cancer in vivo. The effect of high-dose onion flavonoids is similar to that of capecitabine. Onion flavonoids are abundantly available in raw materials, are convenient to extract, and have no toxic side effects. The cancer-suppressive mechanism is further clarified based on these findings, and our results provide new perspective and direction for the prevention and treatment of colorectal cancer.

TG and Lpa levels were significantly decreased in these eight groups in week 3 . This may be associated with the consumption of nutrients during tumor proliferation. However,

\section{Tumor weight after sacrifice}

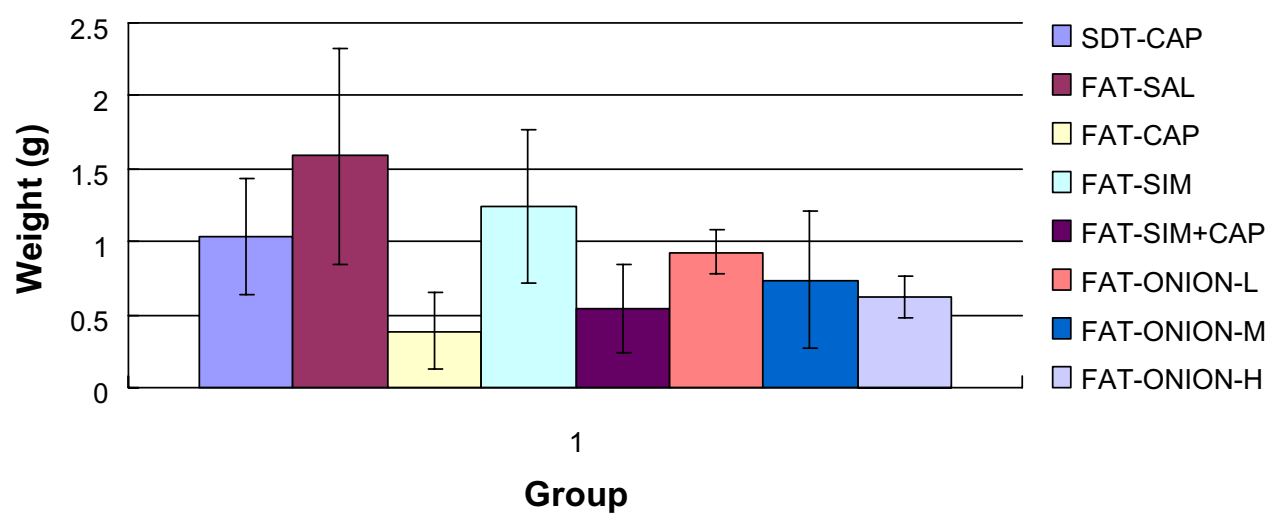

Figure 3 Tumor weight after death. Tumors in the FAT-SAL group showed the most growth, and the growth rate in the FAT-SAL group was significantly faster than that in the SDT-CAP, FAT-CAP, FAT-SIM+CAP, FAT-ONION-L, FAT-ONION-M, and FAT-ONION-H groups. No significant difference was observed between the FAT-SIM group and the FAT-SAL group. The growth rate in the FAT-SIM group was only slightly higher than that in the SDT-CAP and FAT-ONION-L groups, but markedly higher than that in the FAT-CAP, FAT-SIM+CAP, FAT-ONION-M, and FAT-ONION-H groups. The lightest tumor weight was in the FAT-CAP group, and the tumor weight in the FAT-CAP group was lower than that in the FAT-ONION-L $(P<0.05)$, FAT-SIM+CAP $(P>0.05)$, FAT-ONION-M $(P>0.05)$, and FAT-ONION-H $(P>0.05)$ groups. Among the FAT-ONION-L, FAT-ONION-M, and FAT-ONION-H groups, mice in FAT-ONION-L presented the maximum tumor weight, and mice in FAT-ONION-H showed the lightest tumor weight. However, no significant difference was observed among these three groups.

Abbreviations: SDT-CAP, standard diet with capecitabine; FAT-SAL, fat food with saline; FAT-CAP, fat food with capecitabine; FAT-SIM, fat food with simvastatin; FATSIM+CAP, fat food with simvastatin and capecitabine; FAT-ONION-L, fat diet and low-dose onion flavonoids; FAT-ONION-M, fat diet and middle-dose onion flavonoids; FAT-ONION-H, fat diet and high-dose onion flavonoids. 
Table 8 Detection of blood lipid levels 3 and 5 weeks after treatment (week 3 [W3], n=3 $\times 5$; week 5 [W5], n=7 $\times 5$ )

\begin{tabular}{|c|c|c|c|c|c|c|c|c|}
\hline & SDT-CAP & FAT-SAL & FAT-CAP & FAT-SIM & $\begin{array}{l}\text { FAT- } \\
\text { SIM+CAP }\end{array}$ & $\begin{array}{l}\text { FAT- } \\
\text { ONION-L }\end{array}$ & $\begin{array}{l}\text { FAT- } \\
\text { ONION-M }\end{array}$ & $\begin{array}{l}\text { FAT- } \\
\text { ONION-H }\end{array}$ \\
\hline \multicolumn{9}{|l|}{ TC } \\
\hline W3 & $2.10 \pm 0.23$ & $3.80 \pm 0.42$ & $2.76 \pm 1.77$ & $2.94 \pm 0.68$ & $1.39 \pm 0.88$ & $1.45 \pm 1.15$ & $2.26 \pm 1.30$ & $1.47 \pm 0.66$ \\
\hline W5 & $2.65 \pm 1.37$ & $2.74 \pm 1.68$ & $2.44 \pm 1.24$ & $2.57 \pm 1.06$ & $2.57 \pm 1.09$ & $2.55 \pm 1.26$ & $5.27 \pm 1.17$ & $3.83 \pm 2.80$ \\
\hline \multicolumn{9}{|l|}{ TG } \\
\hline W3 & $0.77 \pm 0.08$ & $0.35 \pm 0.12$ & $0.49 \pm 0.04$ & $0.40 \pm 0.07$ & $0.44 \pm 0.13$ & $0.63 \pm 0.53$ & $0.47 \pm 0.20$ & $0.4 I \pm 0.22$ \\
\hline W5 & $0.86 \pm 0.45$ & $0.73 \pm 0.14$ & $0.69 \pm 0.10$ & $0.66 \pm 0.15$ & $0.60 \pm 0.12$ & $0.68 \pm 0.20$ & $1.12 \pm 0.39$ & $0.66 \pm 0.39$ \\
\hline \multicolumn{9}{|l|}{ HDL } \\
\hline W3 & $1.42 \pm 0.33$ & $2.20 \pm 0.30$ & $1.50 \pm 0.92$ & $1.74 \pm 0.60$ & $1.00 \pm 0.52$ & $0.84 \pm 0.73$ & $1.38 \pm 1.17$ & $0.95 \pm 0.53$ \\
\hline W5 & $1.87 \pm 1.13$ & $1.73 \pm 0.85$ & $1.55 \pm 0.89$ & $1.67 \pm 0.93$ & I. $54 \pm 0.78$ & $2.27 \pm \mathrm{I} .4 \mathrm{I}$ & $3.38 \pm 1.04$ & $2.35 \pm 1.86$ \\
\hline \multicolumn{9}{|l|}{ LDL } \\
\hline W3 & $0.39 \pm 0.12$ & $0.47 \pm 0.24$ & $0.44 \pm 0.24$ & $0.37 \pm 0.20$ & $0.20 \pm 0.15$ & $0.21 \pm 0.11$ & $0.21 \pm 0.10$ & $0.28 \pm 0.03$ \\
\hline W5 & $0.35 \pm 0.12$ & $0.42 \pm 0.16$ & $0.30 \pm 0.13$ & $0.37 \pm 0.08$ & $0.36 \pm 0.12$ & $0.67 \pm 0.30$ & $0.62 \pm 0.18$ & $0.47 \pm 0.23$ \\
\hline \multicolumn{9}{|l|}{ Lpa } \\
\hline W3 & $101.00 \pm 14.42$ & $103.00 \pm 55.15$ & $132.00 \pm 12.12$ & $94.00 \pm 11.27$ & $81.00 \pm 9.90$ & $117.67 \pm 7.57$ & $106.67 \pm 2.52$ & $95.33 \pm 9.07$ \\
\hline W5 & $121.83 \pm 12.73$ & $80.57 \pm 35.12$ & $93.83 \pm 32.98$ & $102.00 \pm 27.95$ & $102.00 \pm 9.94$ & $82.50 \pm 30.98$ & $79.67 \pm 18.03$ & $81.00 \pm 23.48$ \\
\hline \multicolumn{9}{|l|}{ apoA } \\
\hline W3 & $\mathrm{I},|4| .4 \mathrm{I} \pm 462.88$ & $455.59 \pm 51.31$ & $433.13 \pm 47.87$ & $419.31 \pm 67.97$ & $844.28 \pm 190.56$ & $519.51 \pm 138.03$ & $374.40 \pm 213.01$ & $139.46 \pm 84.84$ \\
\hline W5 & $956.56 \pm 701.93$ & $555.54 \pm 654.82$ & $431.91 \pm 276.72$ & $427.49 \pm 386.70$ & $397.63 \pm 87.95$ & $362.16 \pm 97.23$ & $371.49 \pm 215.03$ & $279.23 \pm 145.43$ \\
\hline \multicolumn{9}{|l|}{ apoB } \\
\hline W3 & $475.84 \pm 118.90$ & $317.40 \pm 0.00$ & $350.02 \pm 64.95$ & $317.40 \pm 12.11$ & $327.89 \pm 222.42$ & $221.87 \pm 52.46$ & $238.18 \pm 46.54$ & $|24.0| \pm 56.93$ \\
\hline W5 & $284.78 \pm 261.57$ & $218.54 \pm 236.5 \mid$ & $227.55 \pm 165.64$ & $365.37 \pm 82.10$ & $432.32 \pm 89.97$ & $341.28 \pm 65.49$ & $476.72 \pm 139.37$ & $419.50 \pm 32.37$ \\
\hline
\end{tabular}

Abbreviations: SDT-CAP, standard diet with capecitabine; FAT-SAL, fat food with saline; FAT-CAP, fat food with capecitabine; FAT-SIM, fat food with simvastatin; FATSIM+CAP, fat food with simvastatin and capecitabine; FAT-ONION-L, fat diet and low-dose onion flavonoids; FAT-ONION-M, fat diet and middle-dose onion flavonoids; FATONION-H, fat diet and high-dose onion flavonoids; TC, total cholesterol; TG, triglyceride; HDL, high-density lipoprotein; LDL, low-density lipoprotein; Lpa, lipoprotein(a); apoA, apolipoprotein A.

TG levels in FAT-SAL, FAT-CAP, and FAT-SIM+CAP in week 5 were markedly higher than week 3 . These findings indicate that continuous tumor proliferation may gradually increase TG level. These mice were observed for a short time and fed a high-fat diet; therefore, this finding could be further confirmed by excluding the high-fat diet condition. Although it has been reported that abnormal metabolism correlated with the occurrence of colorectal cancer, the causality needs to be clarified. ${ }^{28-30}$ Also, during 3 weeks of capecitabine administration, hypertriglyceridemia was not induced by capecitabine in SDT-CAP or FAT-CAP, as described previously. ${ }^{15,22-24}$ This may relate to the short administration time and limited observation time.

Onion plays a role in decreasing blood fat and protecting heart and blood vessels. ${ }^{31,32}$ In our study, the LDL level significantly increased in SDT-CAP, FAT-SAL, and FAT-CAP, and decreased in FAT-SIM, FAT-SIM+CAP, FAT-ONION-L, FAT-ONION-M, and FAT-ONION-H. This indicates that both simvastatin and onion flavonoids play a role in regulating LDL level. TC and apoB levels in FAT-ONION-L, FAT-ONION-M, and FAT-ONION-H were markedly lower than in FAT-SAL at the beginning and at week 3. The data showed that onion flavonoids can decrease the blood lipid level of animals with abnormal lipid metabolism. Notably, onion flavonoids decrease apoB levels and apoB located on the surface of LDL. Additionally, LDL is generated from very LDL, and contains 50\% endogenous cholesterol, $5 \%$ TG, $20 \%$ protein, and $25 \%$ phospholipid. Cells recognize and absorb LDL by distinguishing apoB. Therefore, increased apoB elevates LDL and TC levels. Our findings suggested that onion flavonoids decrease LDL and TC levels via effectively reducing the apoB level.

In this study, the hyperlipidemia subcutaneous heterotopic colorectal cancer orthotopic transplant model was established. This represents a model for the study of colorectal cancer and abnormal lipid metabolism simultaneously, and it is valuable for the prevention and treatment of colorectal cancer. Onion flavonoids play a role in inhibiting colorectal cancer and decreasing hyperlipidemia. This provides a theoretical foundation for clinical prevention of colorectal cancer by onion and for the treatment and prevention of hyperlipidemia.

\section{Author contributions}

Heiying Jin: Designing the study, analysis and interpretation of data, revising the manuscript, final approval of the version to be submitted and agreement to be accountable for all aspects of the work. 
Yongshan He: Design the study; Drafting the manuscript; Analysis and interpretation of data; Final approval of the version to be submitted; Agreement to be accountable for all aspects of the work. Wei Gong: Analysis and interpretation of data, drafting of the manuscript, final approval of the version to be submitted and agreement to be accountable for all aspects of the work. Chunxia Zhang: Analysis and interpretation of data, revising the manuscript, final approval of the version to be submitted and agreement to be accountable for all aspects of the work. Acheng Zhou: Analysis and interpretation of data, revising the manuscript, final approval of the version to be submitted and agreement to be accountable for all aspects of the work.

\section{Acknowledgment}

The study was funded by the National Nature Science Foundation of China (30572447, 30973837, 81273944).

\section{Disclosure}

The authors report no conflicts of interest in this work.

\section{References}

1. Izzo AA, Capasso R, Capasso F. Eating garlic and onion: a matter of life or death. Br J Cancer. 2004;91(1):194.

2. Galeone $\mathrm{C}$, Pelucchi $\mathrm{C}$, Levi F, et al. Onion and garlic use and human cancer. Am J Clin Nutr. Nov 2006;84(5):1027-1032.

3. Gonzalez CA, Riboli E. Diet and cancer prevention: where we are, where we are going. Nutr Cancer. 2006;56(2):225-231.

4. Jin H, Leng Q, Li C. Dietary flavonoid for preventing colorectal neoplasma (review). Cochrane Database Syst Rev. 2012;8:CD009350.

5. Zhou AC, Jin HY, Tan XZ, et al. Allium cepa extracts inhibit the proliferation of colorectal cancer in vitro. Shi Jie Hua Ren Xiao Hua Za Zhi. 2011;19(19):2011-2015.

6. Yang MH, Rampal S, Sung J, et al. The association of serum lipids with colorectal adenomas. Am J Gastroenterol. 2013;108(5):833-841.

7. Harima S, Hashimoto S, Shibata H, et al. Correlations between obesity/ metabolic syndrome-related factors and risk of developing colorectal tumors. Hepatogastroenterology. 2012;60(124).

8. Lee KH, Park E, Lee HJ, et al. Effects of daily quercetin-rich supplementation on cardiometabolic risks in male smokers. Nutr Res Pract. 2011;5(1):28-33.

9. Movahedian A, Sadeghi H, Ghannadi A, Gharavi M, Azarpajooh S. Hypolipidemic activity of Allium porrum L. in cholesterol-fed rabbits. $J$ Med Food. 2006;9(1):98-101.

10. Wycherley TP, Moran LJ, Clifton PM, Noakes M, Brinkworth GD Effects of energy-restricted high-protein, low-fat compared with standard-protein, low-fat diets: a meta-analysis of randomized controlled trials. Am J Clin Nutr. 2012;96(6):1281-1298.

11. Jin H, Yang Z, Wang J, Zhang S, Sun Y, Ding Y. A superficial colon tumor model involving subcutaneous colon translocation and orthotopic transplantation of green fluorescent protein-expressing human colon tumor. Tumour Biol. 2011;32(2):391-397.

12. Kolinsky K, Zhang YE, Dugan U, Heimbrook D, Packman K, Higgins B. Novel regimens of capecitabine alone and combined with irinotecan and bevacizumab in colorectal cancer xenografts. Anticancer Res. 2009;29(1):91-98.
13. Kodach LL, Jacobs RJ, Voorneveld PW, et al. Statins augment the chemosensitivity of colorectal cancer cells inducing epigenetic reprogramming and reducing colorectal cancer cell 'stemness' via the bone morphogenetic protein pathway. Gut. 2011;60(11):1544-1553.

14. Si ZH, Jin HY. Lipid metabolism abnormalities in patients with colorectal cancer: distribution characteristics and clinical value. Shi Jie Hua Ren Xiao Hua Za Zhi. 2011;19(34):3542-3545.

15. Seminara P, Losanno T, Emiliani A, Manna G. Cancer chemotherapy and cardiovascular risks: is capecitabine-induced hypertriglyceridemia a rare adverse effect? Cardiology. 2010;116(1):42-44.

16. Hata K, Kubota M, Shimizu M, et al. C57BL/KsJ-db/db-Apc mice exhibit an increased incidence of intestinal neoplasms. Int J Mol Sci. 2011;12(11):8133-8145

17. Koya-Miyata S, Arai N, Mizote A, et al. Propolis prevents diet-induced hyperlipidemia and mitigates weight gain in diet-induced obesity in mice. Biol Pharm Bull. 2009;32(12):2022-2028.

18. Coogan PF, Smith J, Rosenberg L. Statin use and the risk of colorectal cancer. J Natl Cancer Inst. 2007;99(1):32-40.

19. Setoguchi S, Glynn RJ, Avorn J, Mogun H, Schneeweiss S. Statins and the risk of lung, breast, and colorectal cancer in the elderly. Circulation 2007;115(1):27-33.

20. Bonovas S, Filioussi K, Flordellis CS, Sitaras NM. Statins and the risk of colorectal cancer: a meta-analysis of 18 studies involving more than 1.5 million patients. J Clin Oncol. 2007;25(23):3462-3468.

21. Lakshminarayana Reddy CN, Vyjayanti VN, Notani D, Galande S, Kotamraju S. Down-regulation of the global regulator SATB1 by statins in COLO205 colon cancer cells. Mol Med Rep. 2010;3(5):857-861.

22. Javot L, Spaëth D, Scala-Bertola J, Gambier N, Petitpain N, Gillet P. Severe hypertriglyceridaemia during treatment with capecitabine. $\mathrm{Br}$ $J$ Cancer. 2011;104(7):1238-1239.

23. Michie CO, Sakala M, Rivans I, Strachan MW, Clive S. The frequency and severity of capecitabine-induced hypertriglyceridaemia in routine clinical practice: a prospective study. $\mathrm{Br} J$ Cancer 2010;103(5):617-621.

24. Schneiders FL, van den Berg HP, Peters GJ, Verheul HM, van der Vliet HJ. Severe toxicity of capecitabine following uncomplicated treatment with 5-fluorouracil/leucovorin. Med Oncol. 2011;28(4):1136-1139.

25. Shimoyama S. Statins are logical candidates for overcoming limitations of targeting therapies on malignancy: their potential application to gastrointestinal cancers. Cancer Chemother Pharmacol. 2011;67(4):729-739.

26. Millen AE, Subar AF, Graubard BI, Peters U, et al. Fruit and vegetable intake and prevalence of colorectal adenoma in a cancer screening trial. Am J Clin Nutr. 2007;86(6):1754-1764.

27. Chen FX, Wei YX, Hong P, He XL, Wang RH, Liu J. Effects of flavonoids extracted from onion by different ways on the proliferation and apoptosis of HCT116 cells. Pharmacol Clin Chin Mater Med. 2008;24(5):36-40.

28. Kim JH, Lim YJ, Kim YH, et al. Is metabolic syndrome a risk factor for colorectal adenoma? Cancer Epidemiol Biomarkers Prev. 2007;16(8): $1543-1546$.

29. Tabuchi M, Kitayama J, Nagawa H. Hyperglycemia and hypertriglyceridemia may associate with the adenoma-carcinoma transition in colorectal epithelial cells. $J$ Gastroenterol Hepatol. 2008;23(6):985-987.

30. Hafez E, Takahashi T, Ogawa H, et al. High susceptibility to zymbal gland and intestinal carcinogenesis in diabetic Otsuka long-evans Tokushima Fatty rats. J Toxicol Pathol. 2011;24(4):187-193.

31. Rao JJ, Jin H, Pang JX, et al. The lipid-lower effect of onion's extraction on hyperlipidemia mice. J Branch Campus First Mil Med Univ. 2004;27(2):103-104.

32. Majewska-Wierzbicka M, Czeczot H. [Flavonoids in the prevention and treatment of cardiovascular diseases]. Pol Merkur Lekarski. 2012;32(187):50-54. Polish. 


\section{Publish your work in this journal}

OncoTargets and Therapy is an international, peer-reviewed, open access journal focusing on the pathological basis of all cancers, potential targets for therapy and treatment protocols employed to improve the management of cancer patients. The journal also focuses on the impact of management programs and new therapeutic agents and protocols on

patient perspectives such as quality of life, adherence and satisfaction. The manuscript management system is completely online and includes a very quick and fair peer-review system, which is all easy to use. Visit http://www.dovepress.com/testimonials.php to read real quotes from published authors.

Submit your manuscript here: http://www.dovepress.com/oncotargets-and-therapy-journal 